\title{
A study of loop source TEM inversion imaging
}

\author{
Wenda Guo ${ }^{a}$, Xi'an Zhu ${ }^{b}$ \\ School of Information and Communication Engineering, Beijing Information Science and Technology \\ University, Beijing 100101, China \\ atcgwd@126.com, bzhuxian1962@163.com
}

Keywords: TEM, all-time apparent resistivity, smoke-ring, approximate inversion.

\begin{abstract}
Based on the forward theory of loop-source TEM, the all-time apparent resistivity is obtained by calculation. Because of the jumping feature in the early-late apparent resistivity when using induced electromotive force, this paper uses transient electromagnetic field to calculate the all-time apparent resistivity, and obtains the continuous and higher precision value. Two methods are used in inversion imaging, one is the smoke-ring, and the other is fast approximate inversion. According to the research, the smoke-ring is easier to achieve, but the fast inversion can reflect the relationship between depth and conductivity better, the result can be a good reference for the research of longitudinal variation of stratum structure.
\end{abstract}

\section{Introduction}

Transient electromagnetic method (TEM), which is also called the time-domain electromagnetic method, has been developing rapidly recent years in China. The geophysical exploration method is widely used in oil and gas exploration, logging, geophysical engineering and other fields. Loop source is the most common TEM detection device, because the device detect the pure secondary field, it has some advantages such as weak terrain effect, strong ability of separating layers, high resolution. In the real project, induced electromotive force generated by the vertical magnetic field can be detected directly, based on which inversion can be done through calculating the early-late apparent resistivity. There is jumping feature in the transition zone of the early and the late apparent resistivity, so all-time apparent resistivity is obtained in forward to avoid the disadvantage.

Many scientists made great contributions to inversion imaging. Smoke-ring inversion was put forward by Nabighian ${ }^{[1]}$ in 1979 . Electromagnetic field made by equivalent electric current ring can be an equivalent substitution of induced eddy current field underground at a certain time. With the lapse of time, the process of current ring downward and outward diffusion can be seen as a series of "smoke ring" which is spit out of the transmitted ring. Direct inversion method was put forward by A G Nekut ${ }^{[2]}$ in 1987.In China, Yan Liangjun made great contribution to fast inversion ${ }^{[3]}$ and rapid resistivity imaging ${ }^{[4]}$.This paper will compare the results of smoke ring inversion and fast approximate imaging inversion and contrast the methods to obtain a better method in a proper situation.

\section{Forward data}

Magnetic response. The analytical expressions for the magnetic field excited by horizontal electric dipole with the time domain step current on a homogeneous conductive half space(with the resistivity $\rho$ ):

$$
h_{z}(x, y)=\frac{I d l y}{4 \pi r^{3}}\left[\left(1-\frac{3}{2 u^{2}}\right) \operatorname{erf}(u)+\frac{3}{u \sqrt{\pi}} e^{-u^{2}}\right]
$$

If $\mathrm{u}$ is large, we consider it early-time:

$$
h_{z}(t)=\frac{I d l y}{4 \pi r^{3}}\left(1-\frac{3}{2 u^{2}}\right)
$$

If $u$ is small, we consider it late-time: 


$$
h_{z}(t)=\frac{I d l y}{4 \pi r^{3}}\left[\frac{8 u^{3}}{\sqrt{\pi}} \llbracket\left(\frac{1}{15}-\frac{u^{3}}{35}+\frac{u^{4}}{126}\right)\right.
$$

Where $I$ is the amplitude of the step current, $d l$ is the length of the dipole, $t$ is the sampling time, $u=\sqrt{\mu_{0} r^{2} / 4 \rho t}, r=\sqrt{x^{2}+y^{2}}$.

We can see that there is great step evolution between early-time response and late-time response, vertical magnetic response is studied to avoid the disadvantage.

$$
H_{z}(\omega)=a I(\omega) \int_{0}^{\infty} \frac{Z^{(1)}}{Z^{(1)}+Z_{0}} J_{1}(\lambda a) J_{0}(\lambda r) d \lambda
$$

Where $\mathrm{A}$ is the radius of the transmitter loop, in which there is step current $I(t)$, using the filter coefficient of Guptasma ${ }^{[5]}$ to do the Hankel transform for the formula (4), then convert it to the time domain through polygonal approximation cosine transform ${ }^{[6]}$, so that the transient electromagnetic response can be obtained.

$$
h_{z}(t) \approx-\frac{2}{\pi t^{2}} \sum_{i=0}^{N} \frac{\operatorname{Im} H_{z}\left(\omega_{i+1}\right) / \omega_{i+1}-\operatorname{Im} H_{z}\left(\omega_{i}\right) / \omega_{i}}{\omega_{i+1}-\omega_{i}} \times\left[\cos \left(\omega_{i} t\right)-\cos \left(\omega_{i+1} t\right)\right]
$$

All-time apparent resistivity. Research of Transient electromagnetic resistivity has great development in China. In 2002,using induction electromotive force to obtain virtual all-time apparent resistivity is put forward by Su Zhu liu; in 2003,Bai Denghai ${ }^{[7]}$ offered a numerical method to calculate all-time apparent resistivity; In 2008, Wang Huajun ${ }^{[8]}$ and Yang Sheng respectively discusses the translation algorithm of apparent resistivity and all-time apparent resistivity formula with consideration of turn-off time. The calculation of the all-time apparent resistivity has gradually become an integral part of ring source TEM inversion. This paper refers to Bai Denghai's definition of the all-time apparent resistivity, when the medium is homogeneous half space, the vertical magnetic field intensity component on the surface can be expressed as follows:

$$
H_{z}\left(\rho_{a}, t\right)=\frac{I_{0}}{2 a}\left[\left(1-\frac{3}{u^{2}}\right) \varphi(u)+\frac{3 \sqrt{2}}{u \sqrt{\pi}} e^{-u^{2} / 2}\right]
$$

There is the kernel function:

$$
Z(u)=\left(1-\frac{3}{u^{2}}\right) \varphi(u)+\frac{3 \sqrt{2}}{u \sqrt{\pi}} e^{-u^{2} / 2}
$$

Where error function is $\varphi(u)=\frac{2}{\pi} \int_{0}^{u} e^{-x^{2}} d x$, in which u can be obtained by dichotomy, and after getting $\mathrm{u}$, the all-time apparent resistivity can be obtained. If $u=\frac{2 \pi a}{\tau}, \tau=\sqrt{2 \pi \rho_{a} t \square 10^{7}}$, then $\rho_{a}=\frac{\mu_{0} a^{2}}{2 u^{2} t}$.

\section{Smoke-ring inversion}

The vertical depth and radius of smoke ring respectively are:

$$
\begin{aligned}
& D_{r}=\frac{4}{\sqrt{\pi}} \sqrt{\frac{t \rho}{\mu_{0}}} \\
& R_{r}=a+2.091 \sqrt{\frac{t \rho}{\mu_{0}}}
\end{aligned}
$$

Where $a$ is the radius of the transmitter loop, $\rho$ is the resistivity of the homogeneous half space, $t$ is sampling time delay, $u$ is the air permeability.

The vertical speed of the smoke ring is: 


$$
v=\frac{2}{\sqrt{\pi}} \sqrt{\frac{\rho}{\mu_{0} t}}
$$

Then we obtain the resistivity:

$$
\rho=\frac{v^{2} \pi t \mu_{0}}{4}
$$

And inversion depth (apparent depth):

$$
H_{r}=0.441 \frac{D_{r j}-D_{r i}}{2}
$$

Where 0.441 is the empirical coefficient.

\section{Rapid approximate imaging}

According to the theory that the secondary induced eddy current can be approximately represented by image source, A. G. Nekut put forward a method that loop source TEM inversion can be done directly. If the vertical magnetic field is $H(t)$, the depth of the image source $D(t)$ can be expressed as follows:

$$
D(t)=R\left\{\left[H(t) / H_{0}\right]^{-2 / 3}-1\right\}^{1 / 2}
$$

Where $H_{0}=\frac{I}{2 R}, I$ is the transmitter current, $R$ is the radius of the transmitter loop. The relationship between the depth of image source and the penetration depth is $D=2 \delta$, the penetration depth of the transient field can be expressed as follows:

$$
\hat{\delta}=\sqrt{\frac{2 t}{\mu_{0} \sigma}}
$$

Where $\sigma$ is all-time apparent conductivity, to correct the error, correction factor is introduced for the correction of the penetration depth. As for the homogeneous half space, the correction factor $F$ is the function of $\hat{\delta} / R$, which means $F(\hat{\delta} / R)=\hat{\delta} / \delta$, when $\hat{\delta} / \delta$ changes from far smaller than 1 to far larger than $1, F$ changes from $1 / \sqrt{2}$ to 1.05.If $H(t)$ is given, the corrected penetration depth can be obtained: $\hat{\delta}=F(\hat{\delta} / R) \square \delta$.We can obtain the zero step conductivity of the transient field: $\sigma^{0}=\frac{2 t}{\mu_{0} \hat{\delta}^{2}}$, and we can also obtain the first order and the second order apparent conductivity:

$$
\begin{aligned}
& \sigma^{1}=\sigma^{0}\left[1+\frac{d \log \sigma^{0}}{d \log \hat{\delta}}\right] \\
& \sigma^{2}=\frac{1}{\mu_{0}} \frac{d^{2} t}{d \hat{\delta}^{2}}
\end{aligned}
$$

Then we can do the inversion through getting the conductivity-depth section.

\section{Comparison between the two methods}

The simulation parameters are as follows: the transmitter current is $10 \mathrm{~A}$,the radius of the transmitter loop is $200 \mathrm{~m}, 41$ sampling points selected in time series.

Table 1 Parameter table of 3-layer H model

\begin{tabular}{ccc}
\hline Layer & Depth(m) & Resistivity $(\Omega \llbracket m)$ \\
\hline 1 & 100 & 120 \\
2 & 80 & 10 \\
3 & & 120 \\
\hline
\end{tabular}


Table 2 Parameter table of 3-layer K model

\begin{tabular}{ccc}
\hline Layer & Depth(m) & Resistivity $(\Omega \llbracket m)$ \\
\hline 1 & 100 & 10 \\
2 & 80 & 100 \\
3 & & 10 \\
\hline
\end{tabular}

Table 3 Parameter table of 4-layer HK model

\begin{tabular}{ccc}
\hline Layer & Depth(m) & Resistivity $(\Omega \square m)$ \\
\hline 1 & 100 & 150 \\
2 & 80 & 15 \\
3 & 100 & 100 \\
4 & & 10
\end{tabular}

Image 1 to image 3 respectively show the comparison of smoke ring inversion, rapid first order and rapid second order, and the fast inversion results are plotted on double logarithmic coordinates. From image 1 we can see that the result of the smoke ring inversion fits the model very well, first order and second order approximate imaging can reflect the change of formation resistivity, but the second order approximate curve is smoother, and more sensitive to the low resistance. From image 2 we can see that the two methods are not sensitive to high resistance responses. From image 3 we can see that the smoke ring inversion depth is not accurate enough, but can reflect formation resistivity very well, first order and second order approximate imaging are better than the smoke ring inversion on resistivity depth accuracy and morphological change, the second order approximate imaging's effect is the best, especially on the inversion accuracy of depth.

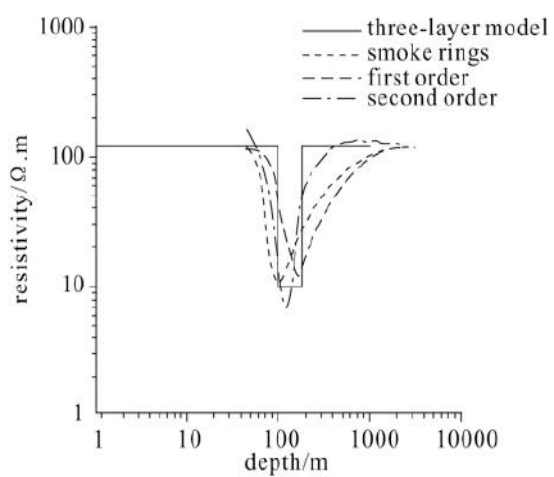

Fig. 1 Two inversion methods for 3-layer $\mathrm{H}$ model Fig. 2 Two inversion methods for 3-layer K

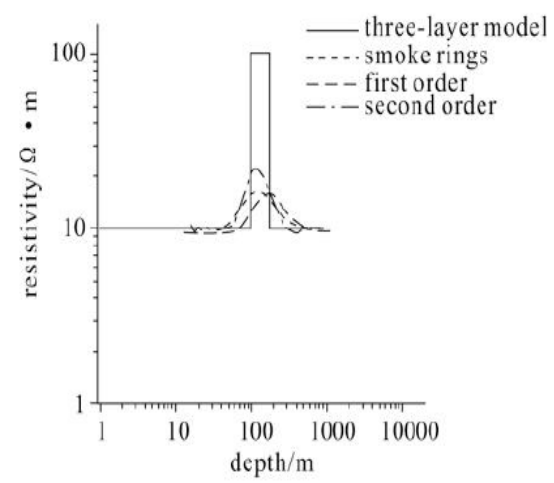
model

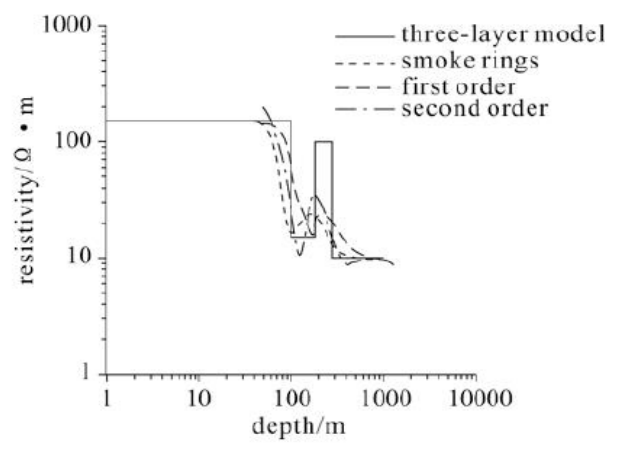

Fig. 3 Two inversion methods about 4-layer HK model

\section{Summary}


In this paper, the magnetic field intensity and all-time apparent resistivity are obtained by forward simulation. Fast imaging part adopts smoke ring inversion and rapid first order and second order approximate imaging to validate the results of the algorithm and model. The results show that they are not sensitive on the high resistance and are sensitive on the low resistivity. The second order approximate imaging effect far better than the first-order approximate imaging both on the resistivity and the depth.

\section{References}

[1]Misac.N.Nabighian, Geophysical investigation-Magnetic method, Geological publishing house,Beijing, 1992.

[2]A.G.Nekut, Direct inversion of time-domain electro-magnetic data, Geophysics. 52(1987) $1431 \sim 1435$.

[3]Yan Liangjun, Hu Wenbao, The estimation and fast inversion of all-time apparent resistivities in long-offset transient electromagnetic sounding, OGP. 34(1999)532 538.

[4]Yan Liangjun, Xu Shizhe, Transient imaging method of fast resistivity sounding and application for the central loop, Coal Geology \& Exploration. 30(2002)58 60.

[5]D.Guptasarma, B.Singh, New digital linear filters for Hankel J0 and J1 transforms, Geophysical Prospecting, 45 (1997)745 762.

[6]Chen Mingsheng, Transient electromagnetic sounding for the electric dipole (one), Coal Geology \& Exploration, 27(1999)55 59.

[7]Bai Denghai, Maxwell, Numerical calculation of all-time apparent resistivity for the central loop transient electromagnetic method, Chinese Journal of Geophysics, 46(2003)697 704.

[8]Wang Huajun, Time domain transient electromagnetism all time apparent resistivity translation algorithm, Chinese Journal of Geophysics. 51(2008)1936 1942. 